\title{
Use of Machine Learning Algorithms in improving the efficiency of flight check-in in Muscat International Airport
}

\author{
Abdullah Saif Mohammed Al Qassabi ${ }^{a}$, Dr. C. Jayakumari ${ }^{\mathrm{a}}$
}

Check-in area in airports is the first step before departure. Usually these areas are very crowded by the passengers who are very sensitive about the time before their flight check-in. Thus, delays while check-in process will have an impact on the passengers in which will have a consequences on the airline company. Delays in check-in process can happen due to various kind of reasons such as airline agent absence where the airline agent is not available at the check-in desk when the time of check-in is started. Artificial intelligence and machine learning are a computer science technologies that can help preventing such problems from occurring. In this paper the machine learning algorithms are studied to detect the staff availability in the check-in desk. The staff is detected by a camera using facial recognition algorithm of machine learning and then analysis are made upon the results found. Whenever there is no staff found the system will notify the airline company and send an expected delay time. The implementation of this solution can be built as a smart based system i.e. based on artificial intelligence and machine learning concepts. All solutions suggested can reduce cost and effect on airline companies and passengers rates respectively.

Keywords: Airport, Check-in, Artificial Intelligence, Machine Learning, Passengers.

\section{Introduction}

Muscat international airport is one of the main and busiest airports around the world since the passengers' rates has increased over the past years. The airport has packed hours in which it gets very crowded in check-in area.

Passengers check-in is a process of enrolling the passengers to their flights that include confirming tickets, dropping baggage and checking traveling documents. The check-in is a time consuming process which causes passengers to be very critical about their time that spent at this area. Delays can happen during flight check-in process which are assignable to different kind of causes.

As the number of passengers is increasing, the chances of delays may increase as well. Airline check-in agent absence is one of different reasons of delays. According to a survey distributed among 100 passengers, $24 \%$ of respondents have been in the check-in area and found no staff available. Preventing the absence of staff will help avoiding any bad outcomes on both the passengers and the airline company. The same survey showed that $75 \%$ of respondents think that using a system will help avoiding delays on passengers' flights.

The machine learning can help solving such issues using facial recognition algorithms that can detect persons' faces and recognize the staff behind the desk. The staff monitoring will start prior to flight opening desk time. If the system start monitoring and found no staff available, it will send notifications to the airline company as an alert along with flight details. The final product that will be delivered is based on artificial intelligence solution. The characteristics of the final product will be a facial recognition system, learning system and analysis system. That said, the objectives of this research are:

1. Build and deliver a machine learning facial recognition system to monitor and detect staff availability.

2. Reduce the delay time and cost due to staff absence on check-in opening time.

3. Measuring the efficiency of facial recognition algorithms in detecting faces.

\section{Literature Review}

Artificial intelligence is a computer engineering started in 1950s and it refers to any system or machine that has intelligence concept (Genç 2019). Different industries have been using $\mathrm{AI}$ in various ways and $\mathrm{AI}$ has become closer to human daily life and styles (Aich 2019). Aviation industry is investing much in AI technologies to make the aviation to align with the needs of airlines and passengers (Sims 2019).

Machine learning is type of AI where the machine more like human in learning and making decisions but faster. Machine learning started in 1980s as a term of how to make the machine think and act as human, then the machine leaning is defined as making the machine act without been programmed (Genç 2019). The main purpose of machine learning is to perform tasks that normal computers or systems cannot do to solve complex problems (Alex and S.V.N. 2009). There are three main types of machine learning; supervised learning, unsupervised learning and reinforcement learning.

Supervised learning is the most used type of machine learning where the data is labeled and the output result is known. It works as the machine is provided with a known data as sample data to recognize the real inputs. Provided that, decision tree, SVM (Support Victor Machine), linear regression and k-nearest neighbors are some of famous supervised learning algorithms (Aich 2019).

Unsupervised learning is the opposite form of the supervised learning, it requires a large number of data set to cluster and associate the data for learning to predict the output result. The data is unlabeled, so based on observation the machine learns to recognize the elements while training. The most used algorithm of unsupervised learning is k-means clustering which is part of clustering techniques, the data are grouped as a cluster then in each iteration the local maxima is found (Genç 2019).

Reinforcement learning is another type of machine learning and it is based on trial and error concept, each time the machine tries to get the correct answer, it get rewarded. In the end, as much as the machine is rewarded the more accurate the result will be. Mainly, it is used for gaming and robots and also in navigations. Q-learning algorithm is one of the most used algorithms for reinforcement learning in which the machine tries to learn from the history while communicating with its environment. 
Facial recognition is biometric system which is used for identifying person using face's nodal points. The facial recognition is most used for security reasons to recognize people for authentication, authorization or identification (Omoyiwola 2018). An advantage of face recognition is that it does not require a direct interaction with the person for recognition. Machine learning uses CNN (Convolutional Neutral Network) algorithms for facial recognition which uses large amount of images in training and learning to classify images (Trigueros et al. 2018). With this in mind, face detection is a technique to determine the human faces in an image frame and it is an important process to perform a face recognition (Tantak et al. 2017).

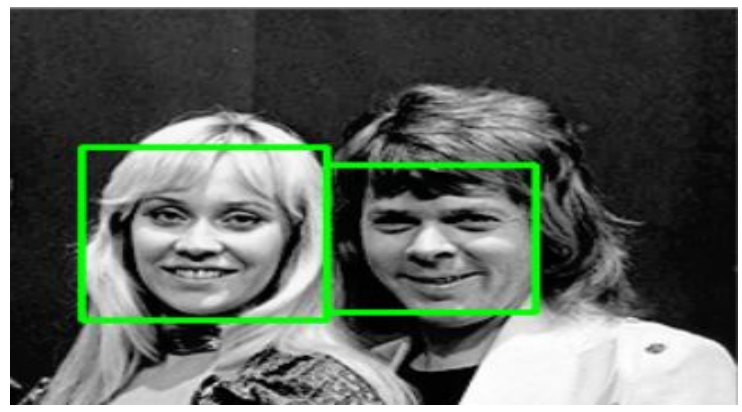

Figure 1. Example of face detection and recognition.

Some organizations have been using face recognition for taking employees attendance, the employee face is given to the camera and then the entrance and out time is registered on coming in and leaving the office (Al-bakeri 2016). Similarly, the same system research is done to register students' attendance in schools to save registration time. The system starts with taking students' pictures labeled with students' names. When the students step in the class, the system start detecting their faces. If the student is present in the class, the record is registered in the database as present. On the other hand, if the student is absent, there will be no record registered so the student is marked is absent (Akshara Jadhav and Tushar Ladhe 2017). Another approach for face recognition is a visitor notification system. Whenever there is a visitor in front of the door, the system detect the visitor's face and send a notification to the house owner with visitor's face. Airports are most critical areas where lot and different kind of people are available in one place, smart systems can help organizing people (passengers) inside the airport. One of USA airports implemented a smart system that uses facial recognition to scan passengers' faces instead of boarding pass for the boarding process ( $\mathrm{CNN}$ newspaper, Street 2019).

\section{Research Methodology}

A research is a study of a problem using a known methods. And there are different types of research methods grouped as qualitative methods which is a collection of information about some knowledge and quantitative methods which is an analysis of data collected as numbers or statistics (McCombes 2019).

Survey and interview are the methods that are used to collect the data regarding the check-in process and staff availability. And they are method types of qualitative and quantitative respectively. Moreover, they are considered as primary data source where the data is the direct source. In addition, the secondary data source is used to describe and improve the primary sources for example use of literature review and book resources.

A survey was distributed over 100 different people who are usually traveling or less travelling though airports. The target people are the ones who are using airport and check-in area. The survey helped getting a numeric and percentage data that provides clear overview of the issue. Not to mention that $76 \%$ of respondents get delayed to their flight due to busy queues while check-in which is quite large percentage. Similarly, $78 \%$ of the respondents think that the long queues will effect on the passengers' flights. The staff absence in check-in desk is a serious problem for the passengers and Airline Company. That said, $82 \%$ of passengers believe that staff availability is required before check-in process starts. Moreover, around 24 out of 100 passengers have been in the check-in desk and there is no staff available. Therefore, most of the passengers with percentage of $75 \%$ agree that staffs availability needs to be detected and $59.6 \%$ think that machine learning and facial recognition significantly will help detecting the availability of the staffs.

Based on the interview, the AMS is an airport management system that has all information about the flights, airlines, check-in counter, staffs, etc. In addition, delays in check-in counter will affect the airline company in which it needs to pay for any extra time the counter is occupied. Also, if the flight take off get delayed the airline company will pay for the extra time of the airplane parking. Number of desks opened is dependent on nature of the flight i.e. crowded flight, type of aircraft, etc. In the end, the need of smart solutions is considered to solve such issues in different areas of the airport like check-in area. All things considered, the machine learning algorithms, especially the facial recognition, is one of the solutions that can help preventing the absence of airline agent during check-in process.

\section{System Design \& Implementation}

System design is the overall view and process flow of the system functionalities and architecture. It is the first step before the implementation where the final product must meet the requirements. The system design consists of both hardware/network design and software design. With this in mind, the hardware and software designs are very basic with high performance. The hardware is any computer that has a video camera attached to it and network adapter or wireless card.

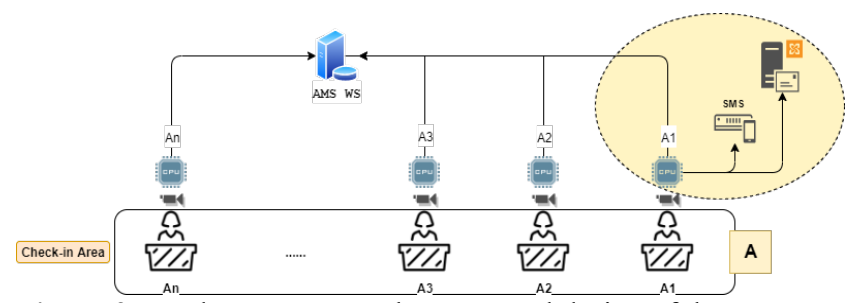

Figure 2. Hardware \& network conceptual design of the system

The system is connected to an online web service which is the AMS web service where all the details about the flights and counters are provided. On the other hand, another connection to an email server (e.g. exchange server) is needed to push email notifications. Similarly, the system has an SMS gateway connection for SMS messages as warning notifications.

The software design of the system shows the process steps on how the system starts and ends the detection of staff availability. The system should identify the staff face and name. If there is a person in front of the desk who is not an airline agent (or ground handler), the system consider it as staff absence. 


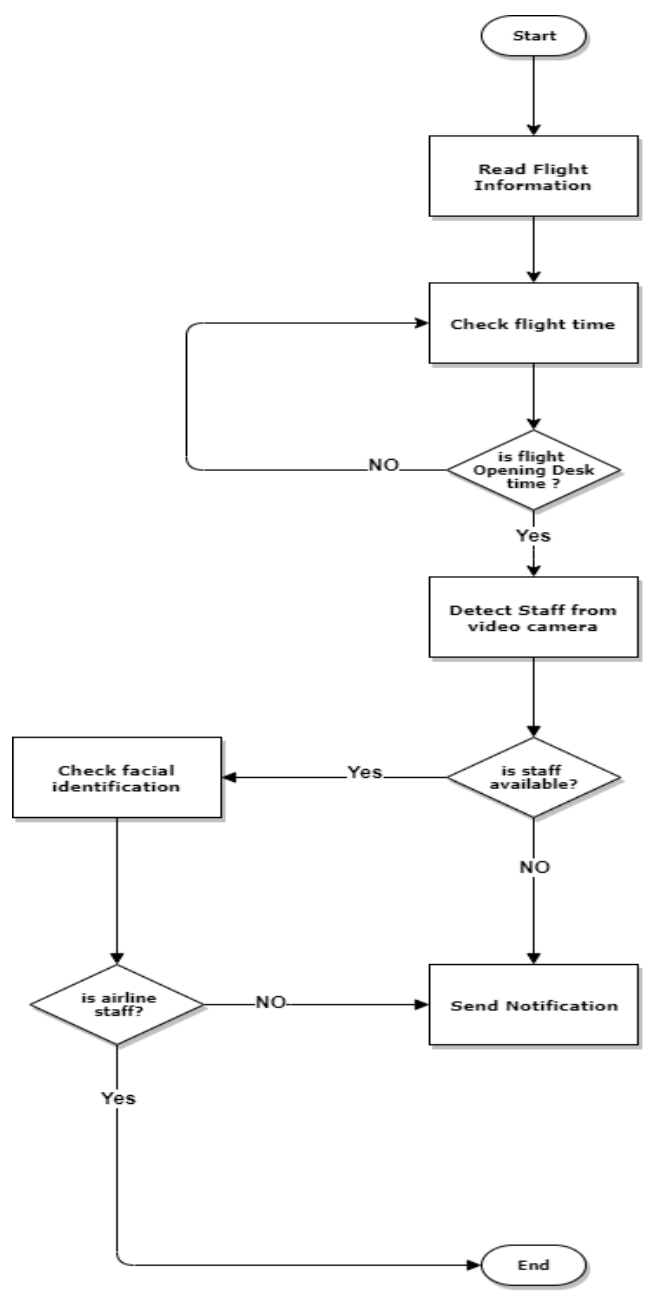

Figure 3. Software conceptual design of the system.

The machine learning development is done by the help of OpenCV framework using python programming language. The OpenCV is an open source computer vision framework which has more than 2000 algorithms which contain functions for face detection and face recognition. Using SVM (support victor machine) algorithm which is a supervised learning algorithm, the machine firstly trained with different labeled pictures as input dataset. The dataset contains two labeled type of pictures, 12 pictures are as known person and 10 as different unknown persons. Then, the machine is tested with pictures of a known person and different unknown persons. The implementation results showed that a percentage between $50 \%$ to $80 \%$ accuracy of face detection. As shown the known person is labeled with the same name when the machine was trained. The other faces are marked as unknown and labeled as unknown. As a result, when there is an unknown staff/person behind the check-in desk, the system consider it as there is no airline agent available.

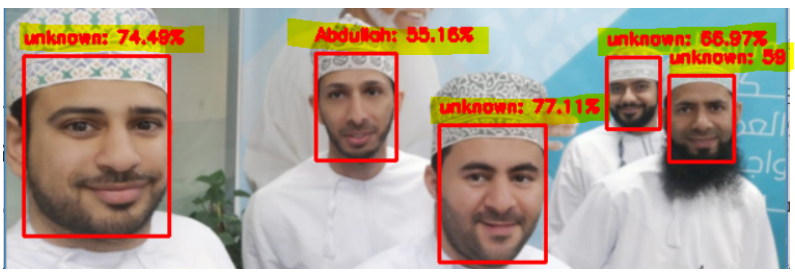

Figure 4. Face recognition test result.
Fourth Middle East College Student Research Conference, Muscat, Sultanate of Oman

All things considered, the final product starts with reading information from the AMS web service. Then, it analyzes the time of opening the counter. According to that the staff detecting will start. If there is no staff available, an email will be sent and the system continue detecting.

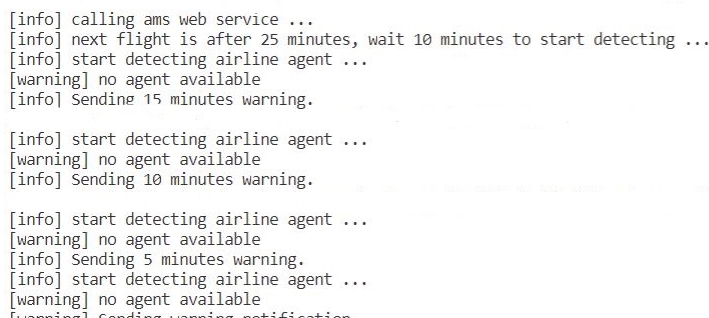

Figure 5. Staff detection system testing.

When the agent is detected, the system should not send any notifications and continue monitoring until the next flight process is started. The system worked successfully as the initial requirements. Also, it can detect faces very fast from different views of the face (i.e. front or sides of the face).

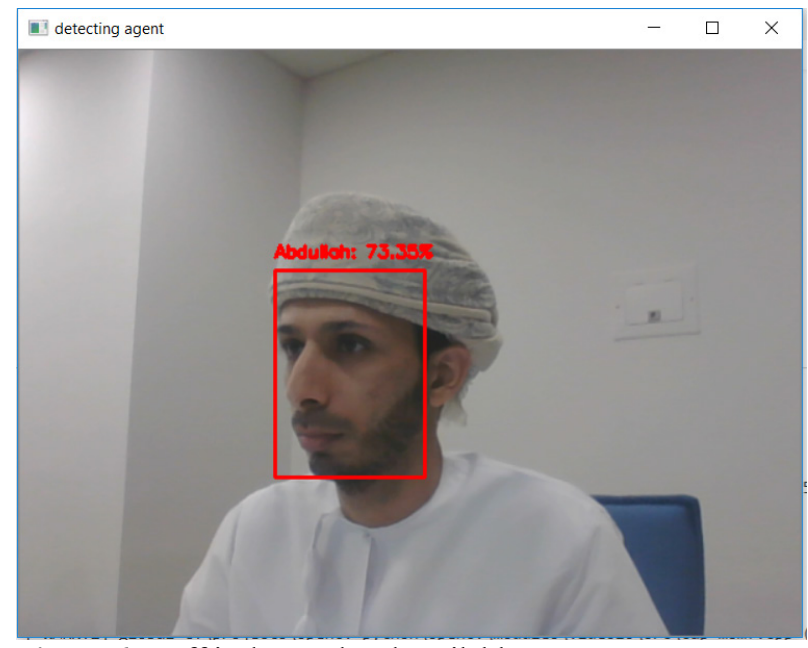

Figure 6. Staff is detected and available.

[info] calling ams web service...

[info] next flight is after 25 minutes, wait 10 minutes to start detecting .. info] start detecting airline agent ...

[info] agent found is ['Abdullah']

Figure 7. Staff is detected and available.

\section{Conclusion}

In this paper, a face recognition system has been implemented using support victor machine (SVM) supervised learning algorithm. Based on OpenCV framework, the system successfully recognized faces in images and videos. The system can identify the agents of the airline company easily. Furthermore, if a new agent joined the company, the agent's photos will be fed to the system as training input. Then the system will extract the person's face and remembers it for further identifications. When the system notice that there is no staff available at the check-in desk, it will push a warning notification as an email to the in charge persons of the considered company. The suggested solution will help preventing any delay consequences since passengers are very sensitive regarding their flights. Artificial intelligence systems are ideal solutions for the airport. Machine learning and facial recognition algorithms help solving issues very fast and accurate. 


\section{References}

Genç, Ö. (2019) Notes on Artificial Intelligence, Machine Learning and Deep Learning for Curious People [online] available from $<$ https://towardsdatascience.com/notes-on-artificialintelligence-ai-machine-learning-ml-and-deeplearning-dl-for-56e 51a2071c2> [30 July 2019]

Aich, A. (2019) What Is Machine Learning and Why It Matters: Everything You Need to Know [online] available from <https://www.knowledgehut.com/blog/datascience/what-is-machine-learning $>\quad[16 \quad$ October 2019]

Sims, N. (2019) Transforming the Future of Airports with Artificial Intelligence, Machine Learning and Generative Design.

Alex, S. and S.V.N., V. (2009) Introduction to Machine Learning. vol. I

Omoyiwola, D. (2018) Machine Learning on Facial Recognition [online] available from $<$ https://medium.com/datadriveninvestor/machinelearning-on-facial-recognition-b3dfba5625a $7>$

Trigueros, D.S., Meng, L., and Hartnett, M. (2018) Face Recognition: From Traditional to Deep Learning Methods. [Online] available from $<$ http://arxiv.org/abs/1811.00116>

Tantak, A., Sudrik, A., Kale, A., and Mehetre, R. (2017) Face Recognition for E-Attendance for Student and Staff. 19 (2), 89-94

Al-bakeri, A.A. (2016) Notification System Based on Face Detection and Recognition: A Novel Approach. (May)

Akshara Jadhav, A.J. and Tushar Ladhe, K.Y. (2017) 'Automated Attendance System Using Face Recognition'. International Research Journal of Engineering and Technology (IRJET) 4 (1), 14671471

Street, F. (2019) How Facial Recognition Is Taking over Airports. [Online] 8 October. Available from $<$ https://edition.cnn.com/travel/article/airportsfacial-recognition/index.html>

McCombes, S. (2019) How to Write a Research Methodology [online] available from $<$ https://www.scribbr.com/dissertation/methodology $>$ [11 November 2019] 